\title{
Massive Hemorrhagic Pericardial Effusion With Cardiac Tamponade as Initial Manifestation of Mixed Connective Tissue Disease
}

\author{
Ashraf Abugroun ${ }^{a}$,, Osama Hallak ${ }^{\mathrm{a}}$, Fatima Ahmed ${ }^{\mathrm{a}}$, Safwan Gaznabi ${ }^{\mathrm{a}}$
}

\begin{abstract}
Mixed connective tissue disease (MCTD) is a distinct entity of connective tissue disorders characterized by overlapping clinical features of various autoimmune diseases along with the presence of antibodies to ribonucleoprotein (anti-RNP). The prevalence of cardiac involvement in MCTD varies from $13 \%$ to $65 \%$ and accounts for approximately $20 \%$ of MCTD related mortality. In this case, we describe an elderly female patient with multiple complaints without a clear etiology on presentation. Echocardiogram revealed severe rapidly accumulating pericardial effusion causing tamponade necessitating pericardial window. Laboratory investigations showed positive ribonucleoprotein antibodies. Biopsy of pericardial tissue revealed fibrinous pericarditis. While pericarditis is commonly associated with MCTD, pericardial tamponade on the other hand is rarely described. This case highlights a very rare complication of the disease. Early recognition, prompt treatment, and regular follow-up with serial echo are essential for treatment.
\end{abstract}

Keywords: Cardiac tamponade; Pericardial window; Mixed connective tissue disease; Ribonucleoprotein antibodies

\section{Introduction}

Mixed connective tissue disease (MCTD) was first described by Sharp et al in 1972 as a distinct entity of connective tissue disease [1]. The disease is characterized by overlapping features characteristic of systemic lupus erythematosus (SLE), rheumatoid arthritis (RA), systemic sclerosis ( $\mathrm{SSc}$ ), and polymyositis/dermatomyositis (PM/DM), along with the presence of antibodies to ribonucleoprotein (anti-RNP) [2]. The disease has a wide spectrum of clinical manifestations due to the involvement of various body organs including the heart.

Manuscript submitted December 8, 2017, accepted December 20, 2017

aAdvocate Illinois Masonic Medical Center, Chicago, IL 60657, USA

bCorresponding Author: Ashraf Abugroun, Advocate Illinois Masonic Medical

Center, 836 W Wellington Ave, Chicago, IL 60657, USA.

Email: ashraf.abugroun@advocatehealth.com

doi: https://doi.org/10.14740/cr654w
Pericarditis is the most common cardiac manifestation of the disease and can affect all layers of the heart. Tamponade on the other hand is a rare manifestation of the disease with limited literature describing such presentation. Here we describe the case of an 81-year-old female who presented with new onset hemorrhagic pericardial effusion requiring drainage and pericardial window. Pericardial tissue biopsy showed fibrinous pericarditis and further serology confirmed positive antiRNP. The patient's condition was controlled with steroids and follow-up echocardiogram showed resolution of the pericardial effusion.

\section{Case Report}

An 81-year-old female presented to the emergency department (ED) complaining of progressive shortness of breath over the preceding few weeks. Other symptoms include weight loss of $30 \mathrm{lb}$ over 5 months, pain in both knees and hips, as well as swelling and numbness in her right-hand fingers. Patient had been following regularly at the cardiology clinic. She had extensive past medical history significant for chronic kidney disease (CKD) stage III, hypertension, coronary artery disease and history of remote myocardial infarction complicated by right ventricular (RV) aneurysm with mural thrombus treated with warfarin therapy, and sick sinus syndrome managed with a pacemaker. She also had remote colon cancer treated with partial colectomy.

On arrival to the ED, she was afebrile, had tachypnea and tachycardia with stable blood pressure. Physical exam revealed fine crackles along the bases and absent pedal edema. She had wide spread hyper pigmented macules and papules over the back and extremities. Initial laboratory workup showed creatinine $2.84 \mathrm{mg} / \mathrm{dL}$, hemoglobin $(\mathrm{Hb}) 8.2 \mathrm{~g} / \mathrm{dL}$, brain natriuretic peptide (BNP) $317 \mathrm{pg} / \mathrm{mL}$ and normal troponin level. Electrocardiogram (EKG) showed ST changes along the inferior leads with low voltage QRS (Fig. 1). Transthoracic echocardiography (TTE) revealed a large circumferential pericardial effusion with tamponade physiology (Fig. 2). Previously seen RV mural thrombus was not identified. Pericardiocentesis was performed with $700 \mathrm{~mL}$ of frankly bloody pericardial fluid drained, and a pigtail catheter was left in place for passive dependent drainage (Fig. 3). Pericardial fluid analysis showed a hemorrhagic exudate without evidence of infection or malignancy based on normal microbiology studies and cytology results. 


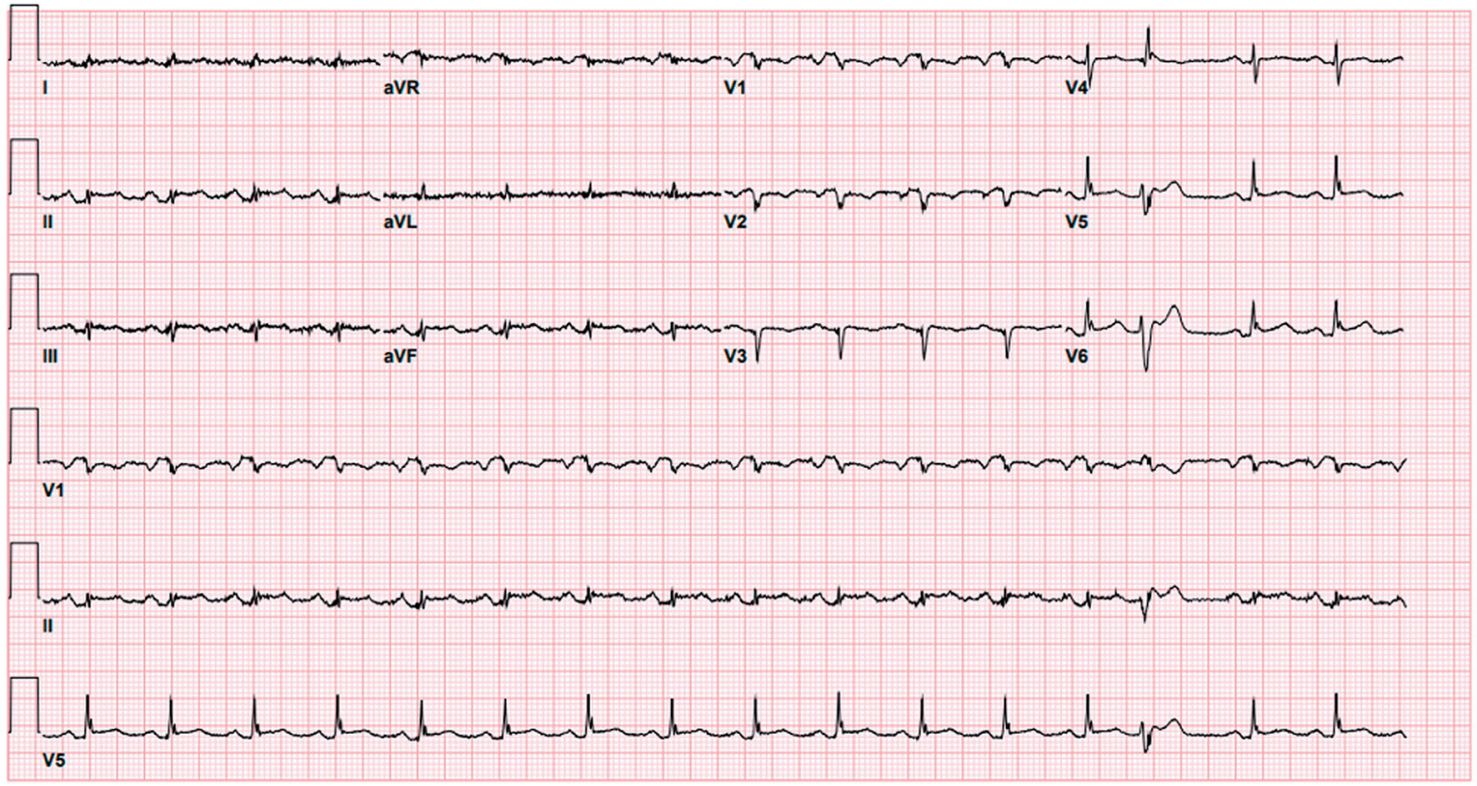

Figure 1. EKG shows sinus rhythm with occasional premature ventricular complexes and low voltage QRS.

Further tests showed an erythrocyte sedimentation rate (ESR) $109 \mathrm{~mm} / \mathrm{h}$, C-reactive protein (CRP) $10.5 \mathrm{mg} / \mathrm{L}$ and normal thyroid function test. She had positive serology for ribonucleoprotein antibodies at 1.9 consistent with a diagnosis of MCTD. Further serology tests were negative for anti-dsDNA, ribosomal P protein, anticentromere Ab, Sjogren's syndrome A and $\mathrm{B}$, Jo1 Ab and Scl-70 Ab.

Computed tomography (CT) scan of the chest, abdomen, and pelvis showed no evidence of malignancy, and tumor markers including CA19-9, CA125 and CEA were negative. Follow-up echocardiogram during admission showed rapidly re-accumulating pericardial effusion. Accordingly, she had a sub-xiphoid pericardial window placed and pericardial tissue biopsy revealed acute and chronic fibrinous pericarditis (Fig. 4). Patient responded to empiric therapy with intravenous prednisone. Prior to discharge, echocardiography showed left ventricular ejection fraction (LVEF) of $30-35 \%$ with only a

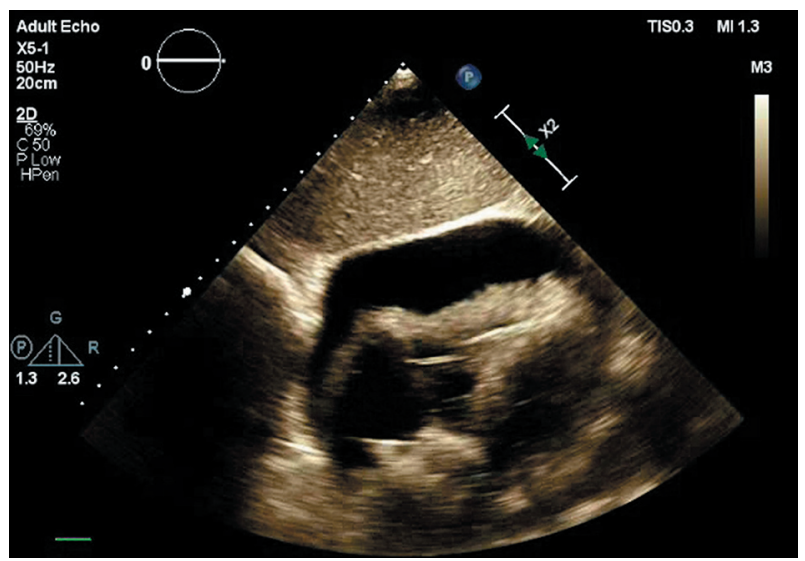

Figure 2. Transthoracic echocardiography, subcostal four chamber view showing diastolic collapse of the RV. trivial pericardial effusion. Given the high risk for recurrence of a pericardial effusion and lack of a mural thrombus, warfarin was held.

\section{Discussion}

MCTD is the first rheumatic disease syndrome that is diag-

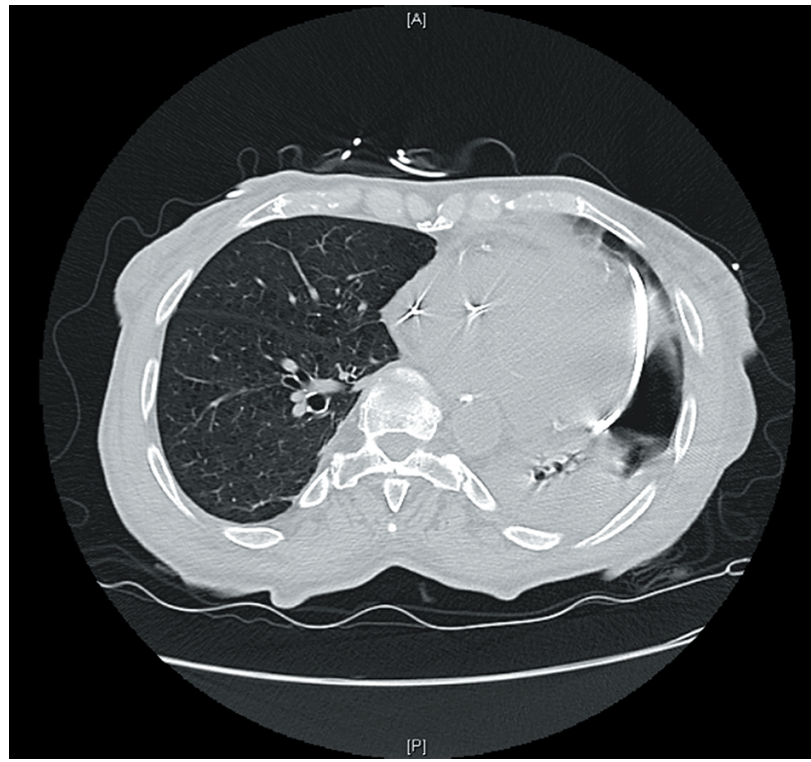

Figure 3. CT scan of the chest shows pigtail catheter coursing within the pericardium and around the anterolateral heart border with tip terminating posterior to the left atrium. Mild to moderate residual pericardial effusion along the inferior heart border. Bilateral pleural effusions, small to moderate on the left and small on the right. Moderate to severe emphysematous changes. 


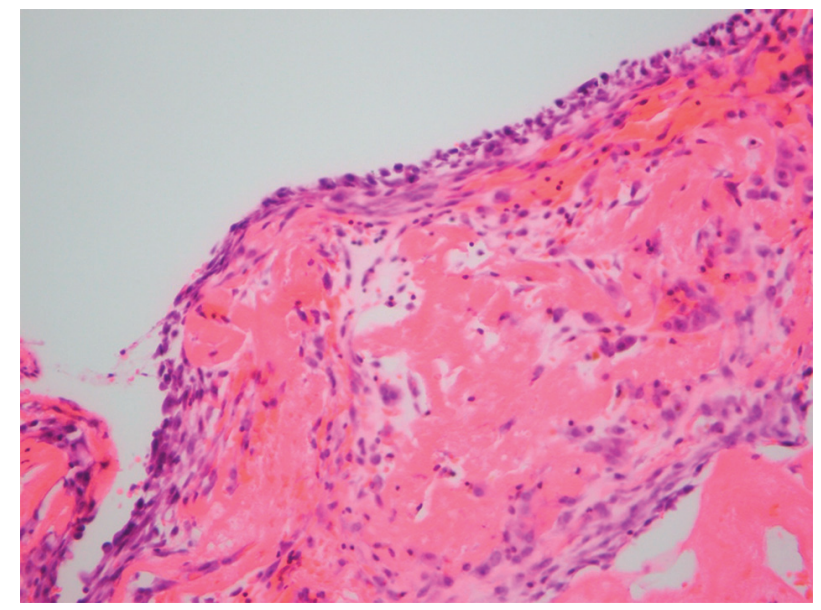

Figure 4. Pericardial tissue biopsy showing reactive mesothelial lining correlated with pericarditis.

nosed based on a serologic test [3]. It is considered a distinct disease entity rather than just a collection of different collagen vascular diseases as it has a special distinctive association with human leukocyte antigen (HLA-DR4) that is different from HLA associated with other connective tissue diseases [4].

Anti-RNP antibodies play a key role in the pathogenesis of MCTD. The mechanism of immune mediated tissue damage and anti-RNP antibody formation in MCTD is multifactorial. Contributing factors include exposure to infectious agents including viruses, modified self-antigens, impaired clearance of potentially immunogenic self-antigens, and decreased lymphocyte tolerance to RNP autoantigens $[5,6]$.

Commonly reported clinical features with the disease include polyarthritis, Raynaud's phenomenon, hand swelling, sclerodactyly, polymyositis, interstitial lung disease, and esophageal dysmotility [3]. Although different diagnostic clinical criteria have been proposed, the disease definition as well as classification remains controversial.

MCTD has variable outcomes ranging from a benign course with favorable therapeutic response to a severe and progressive disease that can be fatal. Approximately one third of patients undergo complete remission and the anti-RNP antibodies completely disappear, while another third of cases ends in a severe progressive course. The most severe complications are usually related to progressive pulmonary hypertension and interstitial lung disease with respiratory failure, cardiac failure, and rarely scleroderma renal crisis with malignant hypertension which can result in fatal outcomes at early age $[3,7]$.

The prevalence of cardiac involvement in MCTD varies from $13 \%$ to $65 \%$ and accounts for approximately $20 \%$ of MCTD related mortality. The most common cardiac manifestation of the disease is pericarditis and cardiac tamponade is rarely described in the natural course of the disease. Other cardiac complications include myocardial dysfunction secondary to pulmonary hypertension as well as accelerated atherosclerosis [8-11]. Non-invasive cardiac tests including EKG and echocardiography are essential for early diagnosis and treatment. Commonly reported findings on EKG include hemi block, bundle branch block, and atrioventricular block.
On the other hand, the most common echocardiographic findings include pericardial effusion and mitral valve prolapse $[10,11]$.

Pericardial effusion results from a wide range of etiologies including infection, malignancies, radiation, metabolic and endocrine causes, as well as autoimmune causes. It can also result from trauma or can be idiopathic. The mechanism of pericardial disease in autoimmune diseases is linked to deposition of immune complexes and inflammatory cells. Pericardial fluid can be either serous, serosanguineous, or hemorrhagic. Tissue biopsy in our patient revealed fibrinous pericarditis, also known as "bread and butter" pericarditis due to its appearance in which the pericardium becomes opaque and granular, and macroscopically resembles two pieces of buttered bread pressed together then pulled apart. The histology shows the deposition of fibrin and leukocytic exudate involving the pericardial leaflets. This is most commonly caused by acute myocardial infarction (MI), trauma/surgery, infection, uremia, neoplasia, and systemic diseases such as in this case [12].

Cardiac tamponade is a medical emergency that results when enough fluid accumulates in the pericardial sack to compress the heart and lead to a decrease in cardiac output and shock. It is diagnosed clinically based on history and physical exam findings, as well as EKG and echocardiography findings. Although our patient had the classic EKG finding of low voltages, this is rarely seen and most patients will only have sinus tachycardia. Echocardiography is the best imaging modality for such cases because it will not only confirm the effusion, but also determine its size and whether it is causing any compromise of cardiac function such as in this case [13].

Pericardiocentesis is the most useful therapeutic procedure for the early management or diagnosis of large, symptomatic pericardial effusion and cardiac tamponade. In hemodynamically unstable patients, the procedure must be done emergently to relieve the pressure on the heart and restore adequate cardiac output. In patients such as ours, who are hemodynamically stable, it is indicated for symptomatic moderate to large effusions non-responsive to medical therapy, when tuberculous, bacterial, or neoplastic pericarditis is suspected, or in the case of a large chronic effusion for longer than 3 months. Of the three main approaches to performing the procedure, fluoroscopy-guided, CT-guided, and echo-guided, we performed a fluoroscopy-guided pericardiocentesis under echocardiographic supervision [14]. Due to the recurrence of the pericardial effusion in our patient, which is a known and common complication of pericardiocentesis, a subxiphoid pericardial window was made which offers a more definitive management. There are currently two techniques for pericardial windows, the subxiphoid such as in this case, which involves approaching the pericardium under the xiphoid process of the sternum, and the thoracotomy technique which comprises access to the pericardium through an incision in the left fifth intercostal space [15].

Treatment of MCTD is largely based on the conventional therapies used for the treatment of autoimmune diseases involved in the condition. Accordingly, therapy is usually individualized based on the disease pattern and organ involvement. Available therapeutic options include corticosteroids, 
methotrexate, antimalarial and cytotoxic agents [16].

\section{Conclusions}

MCTD has a widely variable clinical presentation with distinctive clinical features depending on the end organs involved. Pericarditis is a common cardiac manifestation of MCTD. However, pericardial tamponade is a rare and serious complication of the condition. Early recognition, prompt treatment, and regular follow-up with serial echo are essential for treatment. Future research on MCTD based on large scale sample sizes is needed for better characterization of clinical features of the disease as well as to guide for best treatment modality for each clinical presentation.

\section{References}

1. Sharp GC, Irvin WS, Tan EM, Gould RG, Holman HR. Mixed connective tissue disease--an apparently distinct rheumatic disease syndrome associated with a specific antibody to an extractable nuclear antigen (ENA). Am J Med. 1972;52(2):148-159.

2. Gunnarsson R, Andreassen AK, Molberg O, Lexberg AS, Time K, Dhainaut AS, Bertelsen LT, et al. Prevalence of pulmonary hypertension in an unselected, mixed connective tissue disease cohort: results of a nationwide, Norwegian cross-sectional multicentre study and review of current literature. Rheumatology (Oxford). 2013;52(7):1208-1213.

3. Venables PJ. Mixed connective tissue disease. Lupus. 2006;15(3):132-137.

4. Aringer M, Steiner G, Smolen JS. Does mixed connective tissue disease exist? Yes. Rheum Dis Clin North Am. 2005;31(3):411-420, v.

5. Greidinger EL, Hoffman RW. Autoantibodies in the pathogenesis of mixed connective tissue disease. Rheum Dis Clin North Am. 2005;31(3):437-450, vi.

6. Fujiwara H, Nakamura D, Kukita T, Hamada H, Ozaki A, Matsushita K, Matsumoto T, et al. Immunosuppressive treatment for mixed connective tissue disease may facilitate the development of adult $\mathrm{T}$ cell leukemia/lymphoma in a HTLVI carrier. Intern Med. 2006;45(5):297-301.

7. Lundberg IE. The prognosis of mixed connective tissue disease. Rheum Dis Clin North Am. 2005;31(3):535-547, vii-viii.

8. Arroyo-Avila M, Vila LM. Cardiac tamponade in a patient with mixed connective tissue disease. J Clin Rheumatol. 2015;21(1):42-45.

9. Bezerra MC, Saraiva F, Jr., Carvalho JF, Caleiro MT, Goncalves CR, Borba EF. Cardiac tamponade due to massive pericardial effusion in mixed connective tissue disease: reversal with steroid therapy. Lupus. 2004;13(8):618620.

10. Ungprasert P, Wannarong T, Panichsillapakit T, Cheungpasitporn W, Thongprayoon C, Ahmed S, Raddatz DA. Cardiac involvement in mixed connective tissue disease: a systematic review. Int J Cardiol. 2014;171(3):326-330.

11. Alpert MA, Goldberg SH, Singsen BH, Durham JB, Sharp GC, Ahmad M, Madigan NP, et al. Cardiovascular manifestations of mixed connective tissue disease in adults. Circulation. 1983;68(6):1182-1193.

12. de Souza PM, Dos Santos AM, Paulino EM, Hirth CG, Dornelas CA. "Bread and butter" fibrinous pericarditis. Autops Case Rep. 2016;6(4):5-7.

13. Stashko E, Meer JM. Cardiac Tamponade. [Updated 2017 Feb 7]. In: StatPearls [Internet]. Treasure Island [FL]: StatPearls Publishing; 2017 Jun. Available from: https:// www.ncbi.nlm.nih.gov/books/NBK431090/.

14. De Carlini C, Maggiolini, S. [2017, October 11]. Pericardiocentesis in cardiac tamponade: indications and practical aspects. Retrieved November 28, 2017, from https:// www.escardio.org/Journals/E-Journal-of-CardiologyPractice/Volume-15/Pericardiocentesis-in-cardiac-tamponade-indications-and-practical-aspects.

15. Langdon SE, Seery K, Kulik A. Contemporary outcomes after pericardial window surgery: impact of operative technique. J Cardiothorac Surg. 2016;11(1):73.

16. Kim P, Grossman JM. Treatment of mixed connective tissue disease. Rheum Dis Clin North Am. 2005;31(3):549565 , viii. 